\title{
Ocorrência de enteroparasitas em chupetas de crianças em comunidade carente de Vitória de Santo Antão - PE
}

\author{
Occurrence of enteroparasites in pacifiers of children from a poor community from \\ Vitória de Santo Antão- PE
}

\author{
Brunno Maxwell Lorena Barros ${ }^{1}$; Larissa Isabela Oliveira Souza²; Luís Cláudio Nascimento \\ da Silva ${ }^{1,3}$
}

Resumo: Crianças em idade escolar é o grupo alvo das enteroparasitoses por apresentarem, normalmente, hábitos higiênicos mais precários e/ou ausência de imunidade à re-infecções. $O$ presente trabalho objetivou aferir a frequência de ovos e cistos de enteroparasitas em chupetas de crianças de uma comunidade carente do município de Vitória de Santo Antão - PE e correlacioná-la com os fatores socioeconômicos e higiênicos envolvidos. Foram coletadas 47 chupetas de 42 crianças de ambos os sexos, e aplicados 33 questionários aos seus responsáveis. As chupetas foram coletadas e encaminhadas ao Laboratório de Parasitologia Clínica da Faculdade Pernambucana de Saúde (Recife, PE); onde as chupetas foram lavadas com solução detergente, e em seguida foi realizada a escovação e enxague das mesmas. $O$ líquido resultante foi submetido à centrifugação a $5000 \mathrm{rpm}$. Após 5 minutos o sobrenadante foi desprezado e o sedimento examinado, em duplicata. Das 47 chupetas analisadas $17,02 \%$ estavam parasitadas, sendo os cistos de Entamoeba hystolitica $(37,5 \%)$ e ovos de Ancylostoma sp. $(25 \%)$ os parasitas mais frequentes. Cistos de Entamoeba coli e Giardia lamblia, e ovos de Enterobius vermiculares estiveram presentes em $12,5 \%$ das chupetas. $62,5 \%$ das chupetas parasitadas eram de crianças do sexo masculino. Foi observado correlação do parasitismo das chupetas com fatores higiênico-sanitário da população, reafirmando que é necessária á implantação de trabalho educativo higiênico-sanitário contínuo para conscientização da população.

Palavras-chave: Enteroparasitoses, Crianças, Chupetas.

\begin{abstract}
This study aimed to establish the prevalence of eggs and cysts of intestinal parasites in children's pacifiers in a disadvantaged community in Vitoria de Santo Antão - PE and to correlate this prevalence with socioeconomic and hygienic factors involved. A total of 47 pacifiers were collected from 42 children of both sexes, as well 33 questionnaires were directly applied to the child's guardians during July, 2012. The pacifiers were collected and sent to the Laboratory of Clinical Parasitology of Faculdade Pernambucana de Saúde (Recife, PE), where each pacifier were washed and brushed with detergent solution, and then the resulting liquid was collected and subjected to centrifugation at $5000 \mathrm{rpm}$. After 5 minutes the supernatant was discarded and the pellet examined in duplicate. From all 47 analyzed pacifiers, about $17 \%$ were found parasitized, and the highest incidence was observed for cysts of Entamoeba hystolitica (37.5\%), followed by eggs of Ancylostoma sp. (25\%). Cysts of Entamoeba coli and Giardia lamblia and eggs of Enterobius vermicularis were present in $12.5 \%$ of cases. Furthermore, $62.5 \%$ of parasitized pacifiers were from boys. It was observed a positive correlation between the parasitism of pacifiers and the hygienic-sanitary factors of the population, highlighting that the implementation of hygiene and sanitary educational work for continuous public awareness is needed.
\end{abstract}

Keywords: Enteroparasitosis, Child, Pacifier.

\footnotetext{
${ }^{1}$ Faculdade Pernambucana de Saúde - FPS, Recife, PE, Brasil;

${ }^{2}$ Centro de Pesquisa Aggeu Magalhães - CPqAM-FioCruz, Recife, PE, Brasil;

${ }^{3}$ Universidade Ceuma, São Luís, MA, Brasil.
} 


\section{Introdução}

As parasitoses intestinais são as infecções mais comuns em seres humanos, responsáveis por elevada taxa de morbidade e mortalidade em países em desenvolvimento e subdesenvolvidos. São infecções de importância na saúde pública e seus fatores predisponentes estão vinculados a sub-regiões, os quais incluem falta de higiene, saneamento básico precário, pobreza, desnutrição e baixa escolaridade ${ }^{1}$. A disseminação das formas infectantes dos helmintos intestinais é um fator determinante para a criação e a manutenção de focos endêmicos, dentre os elementos carreadores de ovos e cistos de enteroparasitas estão: resíduos de esgoto mesmo após tratamento ${ }^{2}$, cédulas de dinheiro ${ }^{3}$, vegetais comercializados $^{4,5}$ ou consumidas em escolas $^{6}$, sanitários de escolas ${ }^{7}$, insetos $^{8}$, chupetas de crianças $^{9}$ e transporte público ${ }^{10}$.

Crianças em idade escolar é o grupo alvo das enteroparasitoses por apresentarem, normalmente, hábitos higiênicos mais precários e/ou ausência de imunidade à reinfecções $^{11}$. O parasitismo intestinal nesse grupo de indivíduos torna-se mais relevante pela possibilidade de redução da absorção intestinal acarretando em diarreia crônica, desnutrição e anemia, o que compromete o desenvolvimento físico e intelectual ${ }^{12}$.

Embora no Brasil onde as parasitoses intestinais estejam entre os principais problemas de saúde pública, a investigação parasitológica tem sido amplamente negligenciado, em especial a faixa etária mais acometida, crianças na idade préescolar e escolar. Embasado nessa problemática, o objetivo da presente pesquisa foi de aferir a frequência de ovos e cistos de enteroparasitas em chupetas de crianças de uma comunidade carente do município de Vitória de Santo Antão - PE e correlacionar com fatores socioeconômicos e higiênicos envolvidos.

\section{Material e método}

O estudo foi do tipo experimental transversal, onde foram examinadas 47 chupetas pertencentes a 42 crianças, de ambos os sexos, na faixa etária de zero a sete anos de idade, residentes de uma comunidade carente em Vitória de Santo Antão-PE e cadastrada na Unidade Básica Saúde de Doutor Alvinho. Também foi aplicado um questionário com a finalidade de analisar as condições socioeconômicas, ambientais e higiênicas referentes à população em estudo. Os responsáveis que aceitaram participar da pesquisa assinaram o termo de consentimento livre e esclarecido.

As chupetas foram coletadas nas próprias residências, colocadas em sacos de polietileno devidamente identificados, acondicionadas em caixa de isopor e imediatamente encaminhadas para análises no Laboratório de Parasitologia Clínica da Faculdade Pernambucana de Saúde. Para pesquisa de enteroparasitas segundo a técnica previamente descrita por Dornelles et al. $^{13}$ as chupetas foram lavadas e escovadas com solução detergente de $\mathrm{pH}$ neutro $(500 \mathrm{~mL}$ de solução fisiológica adicionada de duas a quatro gotas de Tween 80) e com auxílio de uma escova de náilon, sendo essas lavadas com hipoclorito e jato d'água antes de cada operação. Após a escovação, a chupeta foi enxaguada com água destilada e o líquido resultante recolhido em um cálice de vidro. Em seguida o líquido resultante 
da lavagem foi transferido para um tubo de centrífuga, e submetido à centrifugação a 5000 RPM/minutos durante 5 minutos. O sobrenadante foi desprezado, o sedimento examinado entre lâmina e lamínula, em duplicata, uma com uma gota do sedimento e outra com a adição de uma gota de solução de Lugol (para diferenciação morfológica das estruturas). As lâminas foram examinadas ao microscópio com objetiva de 10X e, quando encontradas estruturas suspeitas de larvas e ovos de helmintos ou cistos de protozoários, se procedeu à confirmação com objetiva 40X. Uma chupeta controle foi propositalmente contaminada com uma solução fecal positiva com enteroparasitas, a qual sofreu o mesmo processo de lavagem, e que foi posteriormente analisada.

\section{Resultados}

Foram realizadas 47 análises parasitológicas em chupetas de 42 crianças, sendo $28(61,9 \%)$ do sexo feminino e $19(38,1 \%)$ do sexo masculino. Em $17,02 \%$ das chupetas houve presença de parasitas (tabela 1), dessas $62,5 \%$ eram de crianças do sexo masculino.

Tabela 1: Resultado parasitológico das analises de 47 chupetas de crianças de uma comunidade carente do município de Vitória de Santo Antão - PE.

\begin{tabular}{cccc}
\hline Sexo & $\begin{array}{c}\text { Números de } \\
\text { chupetas }\end{array}$ & Amostras positivas & $\%$ \\
\hline Masculino & 19 & 5 & 26,32 \\
Feminino & 28 & 3 & 10,71 \\
\hline Total & 47 & 8 & 17,02 \\
\hline
\end{tabular}

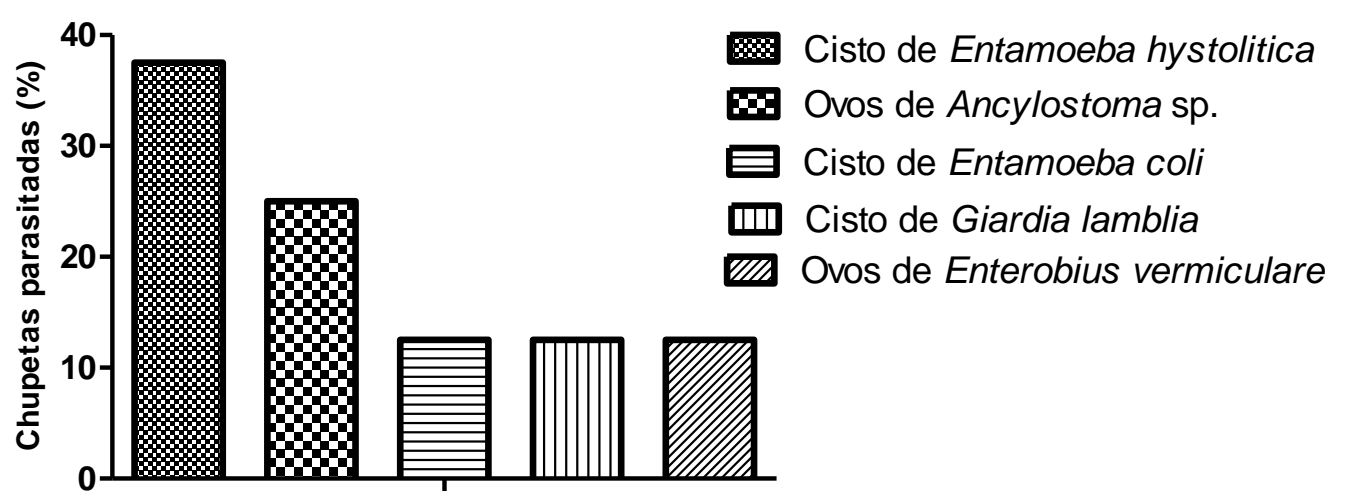

Figura 1: Distribuições dos parasitismos das 47 chupetas de crianças de uma comunidade carente do município de Vitória de Santo Antão - PE. 
Os enteroparasitas mais frequentes foram Entamoeba hystolitica (37,5\%) e Ancylostoma sp. (25\%). Cistos de Entamoeba coli e Giardia lamblia, e ovos de Enterobius vermiculares estiveram presentes em $12,5 \%$ das chupetas (Figura 1). A idade média das crianças usuárias das chupetas com positividade para enteroparasita foi de 3,25 anos de idade (Figura 2).

A idade média dos entrevistados responsáveis pelas crianças usuárias de chupeta foi de 30,6 anos, sendo a maioria do sexo feminino $(96,96 \%)$, $57,56 \%$ tinham apenas 0 ensino fundamental incompleto e 6,1\% eram analfabetos. Todas as crianças moravam em residências do tipo alvenaria e $39,40 \%$ das famílias tinham renda menor que um salário mínimo, a qual provinha do auxilio bolsa Família (Tabela 2).

As residências tinham em média 4,57 moradores, a procedência da água utilizada no lar era de $84,84 \%$ de água encanada, sendo que a maioria $(51,52 \%)$ dos entrevistados não fazia tratamento algum na água antes de utilizá-la para beber e/ou cozinhar. O destino dos dejetos dos domicílios era de $84,84 \%$ para rede pública de esgoto, porém, foram relatados que o mesmo desembocava no rio próximo a comunidade. Todos os domicílios eram cobertos por coleta pública de lixo (Tabela 3).

Em relação a higienização das chupetas foi observado que $12,12 \%$ dos responsáveis não as lavavam, $48,49 \%$ lavam às vezes e $24,24 \%$ só quando as mesmas caiam no chão. Quando questionadas a respeito da água utilizada para a higiene da chupeta, a maioria dos entrevistados $(75,76 \%)$ respondeu que usava a água da torneira. Observou-se também que $36,37 \%$ guardavam as chupetas enrolada na fralda e 30,30\% em cima de móveis (Tabela 3).

As residências tinham em média 4,57 moradores, a procedência da água utilizada no lar era de $84,84 \%$ de água encanada, sendo que a maioria $(51,52 \%)$ dos entrevistados não fazia tratamento algum na água antes de utilizá-la para beber e/ou cozinhar. O destino dos dejetos dos domicílios era de $84,84 \%$ para rede pública de esgoto, porém, foram relatados que o mesmo desembocava no rio próximo a comunidade. Todos os domicílios eram cobertos por coleta pública de lixo (Tabela 3).

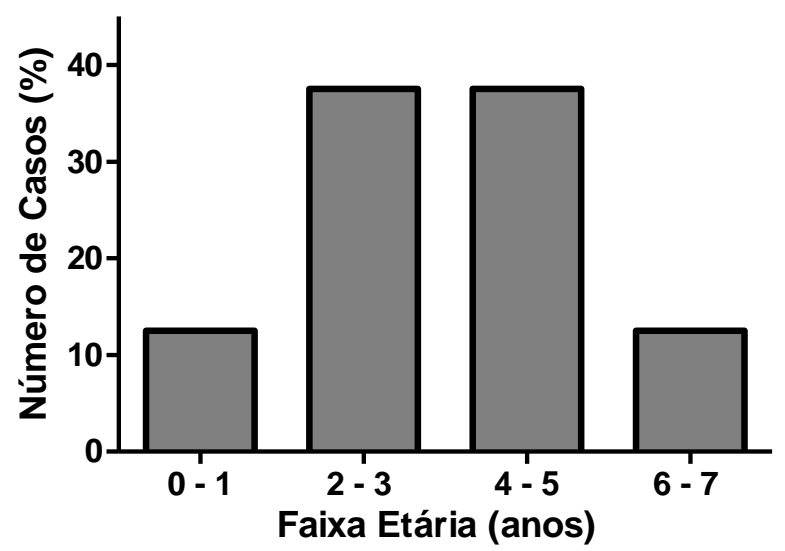

Figura 2: Relação entre a faixa etária/Parasitismo das crianças e chupetas de uma comunidade carente do município de Vitória de Santo Antão - PE. 
Tabela 2: Fatores Socioeconômicos das famílias responsáveis pelas crianças de uma comunidade carente do município de Vitória de Santo Antão - PE.

\begin{tabular}{ccc}
\hline Perguntas & Respostas & $\%$ \\
\hline & Ensino fundamental completo & 0 \\
& Ensino fundamental incompleto & 57,56 \\
Qual o grau de & Ensino médio completo & 12,11 \\
instrução? & Ensino médio incompleto & 24,23 \\
& Ensino superior completo & 0 \\
& Ensino superior incompleto & 0 \\
& Analfabeto & 6,1 \\
Qual o tipo de & Táipa & 0 \\
construção da & Alvenaria & 100 \\
residência? & Barro & 0 \\
& Outros & 0 \\
Qual o tipo do piso & Chão batido & 3,03 \\
da residência? & Alvenaria & 96,97 \\
& Outro & 0 \\
Qual a renda per & Menos que um salário mínimo & 39,40 \\
capita familiar? & Mais que um salário mínimo & 27,27 \\
& Mais que dois salários mínimos & 3,03 \\
& Um salário mínimo & 27,27 \\
& Não soube responder & 3,03 \\
\hline
\end{tabular}

Em relação a higienização das chupetas foi observado que $12,12 \%$ dos responsáveis não as lavavam, $48,49 \%$ lavam às vezes e $24,24 \%$ só quando as mesmas caiam no chão. Quando questionadas a respeito da água utilizada para a higiene da chupeta, a maioria dos entrevistados $(75,76 \%)$ respondeu que usava a água da torneira. Observou-se também que $36,37 \%$ guardavam as chupetas enrolada na fralda e 30,30\% em cima de móveis (Tabela 3).

\section{Discussão}

As enteroparasitoses são um dos problemas básicos na saúde pública de regiões tropicais, podem causar diversos quadros patológicos como diarréia, dores abdominais, distúrbios nutricionais e de comportamento, com isso, crianças parasitadas podem apresentar deficiência no crescimento, na cognição e quadros severos de desnutrição, que podem levar a morte ou a complicações que se estendem por toda a vida do indivíduo ${ }^{10}$.

Estudo anterior realizado em um bairro da periferia de Alfenas - MG, com crianças na mesma faixa etária da presente pesquisa, apresentou percentual semelhante da presença de enteroparasitas em chupetas $(11,63 \%)$, porém com parasitas diferentes (Ascaris lumbricoides e Enterobius vermiculares) ${ }^{9}$.

Em contrapartida, em outra pesquisa realizada com chupetas de escolares em Santa Maria - RS, não houve contaminação de chupetas por 
Tabela 3: Fatores ambientais e higiênicos de 33 responsáveis pelas crianças de uma comunidade carente do município de Vitória de Santo Antão - PE.

\begin{tabular}{|c|c|c|}
\hline Perguntas & Respostas & Valores (\%) \\
\hline $\begin{array}{l}\text { Qual a procedência da } \\
\text { água utilizada no lar? }\end{array}$ & $\begin{array}{l}\text { Água encanada } \\
\text { Água mineral } \\
\text { Água de poço } \\
\text { Pega na casa do vizinho }\end{array}$ & $\begin{array}{c}84,84 \\
0 \\
0 \\
15,16\end{array}$ \\
\hline $\begin{array}{c}\text { Faz algum tratamento } \\
\text { antes de usar a água } \\
\text { para beber e/ou } \\
\text { cozinhar? }\end{array}$ & $\begin{array}{ll}\text { a- } & \text { Nenhum tratamento } \\
\text { b- } & \text { Coloca algum produto } \\
\text { químico } & \\
\text { c- } \quad \text { Fervura } \\
\text { d- } \quad \text { Filtração } \\
\text { e- Água minera } \\
\text { Outro cite }\end{array}$ & $\begin{array}{c}51,52 \\
3.03 \\
9.09 \\
21,21 \\
12.12 \\
3.03\end{array}$ \\
\hline $\begin{array}{l}\text { Qual o destino dos } \\
\text { dejetos? }\end{array}$ & $\begin{array}{ll}\text { a- } & \\
\text { b- } & \text { Fossa } \\
\text { c- } & \text { Rede de esgoto publica } \\
\text { Outro cite }\end{array}$ & $\begin{array}{c}9,09 \\
84,84 \\
6.07\end{array}$ \\
\hline Qual o destino do lixo? & $\begin{array}{ll}\text { a- } & \\
\text { b- } & \text { Coleta pública municipal } \\
\text { c- } & \text { Queima } \\
\text { d- } & \text { Despejo no entorno do rio }\end{array}$ & $\begin{array}{c}100 \\
0 \\
0\end{array}$ \\
\hline $\begin{array}{l}\text { Em relação á chupeta, } \\
\text { ela é lavada antes de ser } \\
\text { dada a criança? }\end{array}$ & $\begin{array}{l}\text { Sim } \\
\text { Não } \\
\text { Ás vezes } \\
\text { Só quando cai no chão }\end{array}$ & $\begin{array}{l}15,15 \\
12,12 \\
48,49 \\
24,24\end{array}$ \\
\hline $\begin{array}{c}\text { Em que tipo de água ela } \\
\text { é lavada? }\end{array}$ & $\begin{array}{ll}\text { b- } & \text { Água da torneira } \\
\text { c- } & \text { Água fervida } \\
\text { d- } & \text { Água filtrada } \\
\text { e- } & \text { Água filtrada e fervida } \\
\text { f- } & \text { Água mineral } \\
\text { g- } & \text { Água e sabão } \\
\text { h- } & \text { Não lava }\end{array}$ & $\begin{array}{l}75,76 \\
12,12 \\
3.03 \\
0 \\
0 \\
0 \\
9,09\end{array}$ \\
\hline $\begin{array}{c}\text { Onde a chupeta é } \\
\text { guardada? }\end{array}$ & $\begin{array}{l}\text { Embaixo do travesseiro } \\
\text { Dentro de uma gaveta } \\
\text { Enrolada na fralda } \\
\text { Em qualquer lugar } \\
\text { Em cima dos móveis } \\
\text { Em um copo com água } \\
\text { Em cima do sofá } \\
\text { Porta chupeta } \\
\text { Na geladeira }\end{array}$ & $\begin{array}{c}3,03 \\
9,09 \\
36,37 \\
3,03 \\
30,30 \\
3,03 \\
3,03 \\
3,03 \\
9,09 \\
\end{array}$ \\
\hline
\end{tabular}


enteroparasitas $^{13}$. Embora metade das chupetas analisadas por esses autores continham micro-organismos do grupo dos coliformes. Esses dados concernem na necessidade de estudos mais detalhados em relação às características das localidades em que as crianças vivem e o tratamento higiênico para com a chupeta, já que os coliformes podem ter origem de contaminação fecal.

Os resultados obtidos chamam a atenção para o fato que, apesar da maioria das chupetas analisadas serem de crianças do sexo feminino, $62,5 \%$ das chupetas parasitadas eram de crianças do sexo masculino, isso ocorre pela suposta maior negligência dos hábitos de higiene deste sexo ${ }^{14}$.

Dentre os enteroparasitas presentes nas chupetas o protozoário mais frequente foi Entamoeba histolytica, a única ameba considerada invasiva, com prevalência elevada nas regiões tropicais, especialmente em comunidades com condições sanitárias inadequadas, e que ocasiona uma acentuada diarreia ${ }^{1}$. Quanto a helminto o Ancylostoma sp. (Ancylostoma duodenale e Necator americanus) foi o mais frequente, possui ampla distribuição geográfica e elevada prevalência, é mais comum em zonas rurais, seu quadro clínico é caracterizado por uma pronunciada anemia e comprometimento pulmonar e intestinal ${ }^{15}$.

No trabalho desenvolvido com gestantes atendidas em um hospital de referência em Recife, a espécie de enteroparasita mais frequente também foi a Entamoeba histolytica ${ }^{16}$. A presença de ovos e cistos encontrados nesta pesquisa foram maiores nas chupetas das crianças na faixa etária de 2 a 5 anos de idade, o que pode ser atribuído ao ganho de autonomia gerando um maior contato com o meio ambiente ${ }^{17}$. Isso fortalece os aspectos negativos associados à utilização de chupetas e devem ser discutidos com os familiares, a fim de racionalizar o seu uso e evitar que esse hábito perdure além dos primeiros meses de vida ${ }^{12,18}$.

As respostas das questões referentes as características socioeconômicas e ambientes traçam um perfil de uma população carente de educação, renda e saneamento básico, fato este, que de acordo com estudo anterior esta associado a alta frequência de enteroparasitoses ${ }^{19}$. Além disto, a maioria dos responsáveis pelas crianças não mostraram ter os cuidados adequados com a higienização das chupetas.

Este trabalho corrobora com outros dados ${ }^{9}$, mostrando que as chupetas são fontes potencias de contaminação, em especial para crianças com intenso contato com o solo e que vivem em comunidades carentes com saneamento básico inadequado. Nessas populações, os enteroparasitas encontram seu grupo alvo preferido para infecção, as crianças em idade pré-escolar e escolar, 0 que caracteriza um importante problema social.

\section{Conclusão}

Com os resultados obtidos conclui-se que a alta frequência de enteroparasitas nas chupetas analisadas pode estar relacionada a fatores determinantes como: a falta de informação, hábitos higiênicos, cuidado com a água e a classe social. Tudo isso provavelmente se deve ao difícil acesso a informação sobre os assuntos. Com isso, é importante à conscientização da população através de ações profiláticas e não só 0 tratamento medicamentoso. Alem de trabalhos sócio-educativos e higiênicosanitários contínuos em escolas e comunidades carentes, levando em 
conta as particularidades culturais de cada região, almejando sempre a melhora da qualidade de vida da população.

\section{Referências}

1. Andrade EC, Leite ICG, Rodrigues VO, Cesca MG. Parasitoses Intestinais: Uma revisão sobre seus aspectos sociais, epidemiológicos, clínicos e terapêuticos. Revista A. P. S. 2010;13(2):231-240.

2. Almeida AF, Onani KAA, Segura-Munoz SI, Santos VM, Takayanagui AMM. Adaptação ao Método de Ritchie para diagnóstico de Helmintos e Protozoários em amostras de lodo de esgoto com minimização de produtos químicos. Mundo saúde 2009;33(4):427-432.

3. Sudré AP, Franco BOP, Zaniboni B, Gonçalves DS, Santos FLA, Branco LG, Guerra RS, Neiva RC, Brener B. Estudo da contaminação de moedas e cédulas de dinheiro circulantes na cidade de NiteróiRJ. Rev. Patol. Trop. 2012;41(4):465-470.

4. Freitas AA. Avaliação
parasitológica de alfaces (Lactuca ativa) comercializadas em freiras livres e supermercados do municipio de Campo Mourão, Estado do Paraná. Acta Sci. Biol. Sci. 2004;26:381-384.

5. Falavigna DLM, Freitas BR, Melo GC, Nishi L, Araújo SM, Vigna-Guilherme AL. Qualidade de hortaliças comercializadas no noroeste do Paraná, Brasil. Parasitol. Latinam. 2005;60:144 $-149$.
6. Coelho LMPS, Oliveira SM, Sá $\mathrm{MH}$, Milman A, Karasawa KA, Santos RP. Detecção de formas transmissíveis de enteroparasitas na água e nas hortaliças consumidas em comunidades escolares de Sorocaba, São Paulo, Brasil. Rev. Soc. Bras. Med. Trop. $2001 ; 34: 479-482$.

7. Coelho LMPS, Adair Sobrinho T, Oliveira SM, Ikegami MT, Yoshizumi AM, Nakamoto AYK, Brotto SA, Felberg S, Maiorano MR. Ovos e larvas de helmintos nos sanitários de pré-escolas municipais de Sorocaba, SP e suas freqüências nas fezes das crianças. Rev. Soc. Bras. Med. Trop. 1999;32:647-652.

8. Thyssen PJ, Moretti TC, Ueta MT, Ribeiro OB. Papel de insetos (Blattodea, Diptera e Hymenoptera) como possíveis vetores mecânicos de helmintos em ambiente domiciliar e peridomiciliar. Cad. Saúde Pública 2004;20:1096-1102.

9. Pedroso RS, Siqueira RV. Pesquisa de cistos de protozoários, larvas e ovos de helmintos em chupetas. J. Pediat., 73: 21-25, 1991.

10. Murta FL, Massara CL. Presença de ovos de helmintos intestinais em ônibus de transporte público em Belo Horizonte - Minas Gerais, Brasil. Rev. Patol. Trop. 2009;38:207-212.

11. Uchôa CMA. Parasitoses intestinais: prevalência em creches comunitárias da cidade de Niterói, Rio de JaneiroBrasil. Rev. Inst. Adolfo Lutz. 2001;60(2):97-101. 
12. Pinheiro RO. Ocorrência de parasitas entre crianças do préescolar de duas escolas em Vassouras, RJ. Rev. Bras. Farm. 2007;88:98-99.

13. Dornelles EVF, Vizzoto BS, Roggia I, Santos RCV. Condições ParasitológicasSanitárias de Chupetas de Crianças em Comunidades Carentes de Santa Maria-RS. NewsLab. 2006;76:142-156.

14. Murta FL, Massara CL. Presença de ovos de helmintos intestinais em ônibus de transporte público em Belo Horizonte - Minas Gerais, Brasil. Rev. Patol. Trop. 2009;38:207-212.

15. Nkrumah B, Nguah SB. Giardia lamblia: a major parasitic cause of childhood diarrhoea in patients attending a district hospital in Ghana. Parasit Vectors. 2011;4:163.

16. Belloto MVT, Santos Jr JE, Macedo EA, Ponce, A, Galisteu KJ, Castro E, Tauyr LV, Rossit ARB, Machado RLD. Enteroparasitoses numa população de escolares da rede pública de ensino do Município de Mirassol, São Paulo, Brasil. Rev. Pan-Amaz. Saúde. 2011;2(1):37-44.

17. Souza AI, Ferreira LOC, Batista Filho M, Dias MRFS. Enteroparasitoses, Anemia e Estado Nutricional em Grávidas Atendidas em Serviço Público de Saúde. Rev. Bras. Ginecol. Obstes. $2001 ; 24(4): 253-259$.

18. Oliveira VF, Amor ALM. Associação entre a ocorrência de parasitos intestinais e diferentes variáveis clínicas e epidemiológicas em moradores da comunidade Ribeira I, Araci, Bahia, Brasil. Rev. Bras. Anál. Clin. 2012;44(1):15-25.

19. Berne AC, Scaini CJ, Vilela MM, Pepe MS, Haupenthal LE, Gatti F, Berne MEA. Presença de coccídios e outros enteroparasitos em uma população de crianças no município de Rio Grande, Rio Grande do Sul, Brasil. Rev. Patol. Trop. 2012;41(1):93-96. 